\title{
Radical Addition of Perfluorinated Alkyl Iodides to Multi- Layered Graphene and Single-Walled Carbon Nanotubes
}

\author{
Christopher E. Hamilton, Jay R. Lomeda, Zhengzong Sun, James M. Tour ( $\varangle)$, and Andrew R. Barron ( $\varangle)$ \\ Richard E. Smalley Institute for Nanoscale Science and Technology, Department of Chemistry and Department of Mechanical Engineering \\ and Materials Science, Rice University, Houston, Texas 77005, USA \\ Received: 16 October 2009 / Revised: 13 November 2009 / Accepted: 18 November 2009 \\ (C) The Author(s) 2010. This article is published with open access at Springerlink.com
}

\begin{abstract}
A simple one-pot reaction that serves to functionalize graphite nanosheets (graphene) and single-walled carbon nanotubes (SWNTs) with perfluorinated alkyl groups is reported. Free radical addition of 1-iodo- $1 \mathrm{H}, 1 \mathrm{H}, 2 \mathrm{H}$, $2 \mathrm{H}$-perfluorododecane to ortho-dichlorobenzene suspensions of the carbon nanomaterial is initiated by thermal decomposition of benzoyl peroxide. Similarly, UV photolysis of 1-iodo-perfluorodecane serves to functionalize the carbon materials. Perfluorododecyl-SWNTs, perfluorododecyl-graphene, and perfluorodecyl-graphene are characterized by infrared (IR) and Raman spectroscopy, X-ray photoelectron spectroscopy (XPS), thermogravimetric analysis (TGA), and atomic force microscopy (AFM). The products show enhanced dispersability in $\mathrm{CHCl}_{3}$ as compared to unfunctionalized starting materials. The advantage of this one-pot functionalization procedure lies in the use of pristine graphite as starting material thereby avoiding the use of harsh oxidizing conditions.
\end{abstract}

\section{KEYWORDS}

Nanotube, single-walled carbon nanotube, graphene, radical addition

\section{Introduction}

Owing to the desirable physical and electronic properties of fullerenes and related materials, research on carbon nanomaterials has been extraordinarily active. Single-walled carbon nanotubes (SWNTs) have been studied increasingly over the past decade and have overshadowed the other polymorphs of carbon [1]. However, graphite has lately enjoyed a renaissance due to the isolation of individual graphene layers [2]. One of the most difficult obstacles in working with carbon nanomaterials is their insolubility due to large van der Waals bundling forces. Efforts toward separating individual nanotubes or graphene nanosheets have focused on either (1) non-covalent dispersion agents such as surfactants, or (2) covalent functionalization wherein the materials are chemically derivatized to permit solubility. Covalent methods can be advantageous in that they often provide more robust materials [3].

Advances in organo-fluorine chemistry have produced many technologically relevant materials. Polytetrafluorethylene (PTFE) materials have found numerous commercial applications resulting from their hydrophobicity (GoreTex) and tribological properties (Teflon). In addition, so-called fluorous phase chemistry has been an active area of research with wide-ranging applications including homogeneous catalysis. By attaching perfluorinated alkyl chains to ligands, homogeneous catalyst complexes can be made

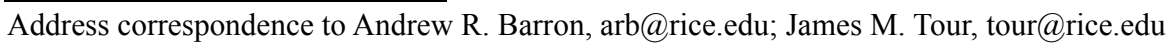


soluble in fluorous solvents (which are immiscible with both water and conventional organic solvents). Fluorous catalysts can then be easily separated from reaction products in biphasic systems [4].

As part of our ongoing effort to attach catalyst particles to SWNTs [5] for seeded growth [6] we investigated the functionalization of SWNTs with perfluorinated alkyls $\left(R_{F}\right)$ in order to disperse the products in fluorous solvents. While there is limited literature concerning perfluorinated alkylation of carbon nanomaterials [7], Hirsch and coworkers have derivatized SWNTs with perfluorooctyl groups via UV photolysis of perfluorooctyl iodide [8]. However, the resulting $R_{\mathrm{F}}$-SWNTs showed no solubility enhancement, and product characterization was limited to ${ }^{19} \mathrm{~F}$ nuclear magnetic resonance (NMR). In addition the Rao group coupled perfluoroundecylamine to SWNT carboxylic acid groups via amide linkages [9]. Their product had a low level of functionalization, however due to the fact that coupling is limited to oxidized SWNT ends and defect sites. Later, the same group reported selective fluorous tagging of metallic SWNTs by diazonium functionalization. Most recently, Pulikkathara et al. perfluorinated alkyl-derivatized SWNTs using elemental fluorine [10]. Clearly, this method is not convenient, as it requires specialized equipment and hazardous reactants.

Billups and coworkers have used benzoyl peroxide to produce phenyl radicals which either phenylate SWNTs directly or generate alkyl radicals from iodides $[11,12]$. This approach provides a simple one-pot reaction that should be amenable to perfluorinated alkyl iodides, with one caveat: perfluorinated alkyl iodides are highly unstable. In fact, in Hirsch's report, UV illumination was sufficient to initiate radical formation [8]. Leaving a two-methylene "spacer" between the fluorocarbon chain and iodine mitigates this extreme instability, however. The resultant 1-iodo- $1 \mathrm{H}, 1 \mathrm{H}, 2 \mathrm{H}, 2 \mathrm{H}$-perfluoroalkane derivatives have stabilities on par with normal alkyl iodides.

As graphite is a robust, thermally stable material that already has lubricant applications, we thought it desirable to functionalize graphene with fluorinated alkyl groups. There are dozens of patents and publications in the literature pertaining to graphite and carbon nanotube-PTFE composite materials.
These materials have been examined for numerous applications and their tribological properties are well studied. However, fabrication of all these composites employs simple physical mixing of carbon materials into polymer matrices and is often plagued by phase segregation problems. To the best of our knowledge, there exists no precedent for the formation of covalent fluorocarbon-nanocarbon hybrid lubricant materials. These materials could lead to vastly improved thermal and tribological properties of fluorocarbonbased lubricant and polymer products. With this goal in mind, we have successfully used radical alkylation chemistry to functionalize SWNTs and multi-layered graphene with perfluoroalkyl groups.

\section{Experimental}

Solvents including 1,2-dichlorobenzene (ODCB) were purchased from Fisher Scientific and were used as received. Benzoyl peroxide was purchased from Sigma-Aldrich and was purified by recrystallization from $\mathrm{CHCl}_{3} /$ methanol [13]. 1-Iodo- $1 \mathrm{H}, 1 \mathrm{H}, 2 \mathrm{H}, 2 \mathrm{H}-$ perfluorododecane was purchased from Alfa Aesar and used as received. 1-Iodoperfluorodecane was purchased from TCI America and used as received. Single-walled carbon nanotubes (HiPco process) were obtained from the Carbon Nanotube Laboratory at Rice University (Houston, TX), and were purified [14] and etched [15] by literature procedures. ODCBgraphene dispersions were prepared using expanded graphite (Supra Carbonic, LLC) or microcrystalline graphite (Sigma-Aldrich) according to our prior report [16]. Sonication refers to bath sonication using a Cole-Parmer Ultrasonic Processor with a Model CV33 probe at room temperature. The following abbreviations are employed in describing the products: $\mathrm{G}=$ graphene, $\mathrm{R}_{\mathrm{F}}-\mathrm{D}=$ perfluorodecyl, and $\mathrm{R}_{\mathrm{F}}-\mathrm{DD}=$ perfluorododecyl.

UV photolysis reactions were performed using a Rayonet RPR-100 photochemical reactor $\left(\lambda_{\max }=254 \mathrm{~nm}\right)$ with a cooling fan and magnetic stirrer. Fourier transform infrared (FTIR) spectra were collected on a Nicolet Magna 760 spectrometer. FTIR samples were prepared by mixing samples with $\mathrm{KBr}$ before pressing into pellets. Raman data were obtained using a Renishaw Raman microscope with a 514 nm (graphene 
materials) or $785 \mathrm{~nm}$ (SWNT materials) laser excitation source. X-ray photoelectron spectroscopy (XPS) data were acquired on a Physical Electronics, Inc. PHI Quantera instrument with a monochromated Al-Ka X-ray source. Atomic force microscopy measurements were obtained using a Digital Instruments NanoScope III a scanning probe microscope in tapping mode. Atomic force microscopy (AFM) samples were spincoated onto freshly cleaved mica. Thermogravimetric analyses (TGAs) analyses were performed on a Seiko I TG/DTA 200 instrument, under an argon flow of 15-20 sccm, using a ramp temperature of $5{ }^{\circ} \mathrm{C} / \mathrm{min}$.

\section{$1.1 \quad R_{\mathrm{F}}$-DD-SWNTs}

In a typical experiment, SWNTs (20 mg) were dispersed in ODCB $(30 \mathrm{~mL})$ by sonication in a Schlenk flask. Benzoyl peroxide $(0.4 \mathrm{~g}, 1.6 \mathrm{mmol})$ and 1-iodo- $1 \mathrm{H}$, $1 \mathrm{H}, 2 \mathrm{H}, 2 \mathrm{H}$-perfluorododecane $(2.7 \mathrm{~g}, 4.0 \mathrm{mmol})$ were added to the SWNT dispersion. The reaction mixture was stirred at $80^{\circ} \mathrm{C}$ for $24 \mathrm{~h}$ under Ar. After cooling to room temperature, $\mathrm{CHCl}_{3}(30 \mathrm{~mL})$ was added and the mixture was vacuum filtered through an alumina membrane $(0.02 \mu \mathrm{m}$ pore size $)$. The product was suspended in $\mathrm{CHCl}_{3}$ by bath sonication and filtered again. Sonication and filtration were repeated twice more to ensure removal of all excess reactants. Finally the filter cake was washed with more $\mathrm{CHCl}_{3}$ and then dried under vacuum. Typical yields were $18-20 \mathrm{mg}$ of the desired product.

\section{$1.2 \quad R_{\mathrm{F}}-\mathrm{DD}-\mathrm{G}$}

In a typical experiment, an ODCB-graphene [19] suspension ( $30 \mathrm{~mL}$ of $0.03 \mathrm{mg} / \mathrm{mL}$ ) was added to a Schlenk flask along with benzoyl peroxide $(0.4 \mathrm{~g}$, $1.6 \mathrm{mmol}$ ) and 1-iodo- $1 \mathrm{H}, 1 \mathrm{H}, 2 \mathrm{H}, 2 \mathrm{H}$-perfluorododecane $(2.7 \mathrm{~g}, 4.0 \mathrm{mmol})$. The reaction mixture was stirred at $80{ }^{\circ} \mathrm{C}$ for $24 \mathrm{~h}$ under Ar. After cooling to room temperature, $\mathrm{CHCl}_{3}(30 \mathrm{~mL})$ was added and the mixture was vacuum filtered through an alumina membrane ( $0.02 \mu \mathrm{m}$ pore size). The solid was suspended in $\mathrm{CHCl}_{3}$ by probe sonication and filtered. Sonication and filtration were repeated twice more to ensure removal of all excess reactants. Finally, the filter cake was washed with additional $\mathrm{CHCl}_{3}$ and then dried in vacuo. Typical yields were $700-800 \mu \mathrm{g}$ of the desired product.

\section{$1.3 \quad R_{F}-D-G$}

In a typical experiment, an ODCB-graphene suspension $(30 \mathrm{~mL}$ of $0.03 \mathrm{mg} / \mathrm{mL}$ ) was added to a round-bottom flask with 1-iodoperfluorodecane (500 $\mathrm{mg}, 0.8 \mathrm{mmol}$ ). The reaction mixture was stirred at room temperature under UV illumination for $10 \mathrm{~h}$. $\mathrm{CHCl}_{3}$ was added $(20 \mathrm{~mL})$ and the mixture was vacuum filtered through an alumina membrane $(0.02 \mu \mathrm{m}$ pore size). The solid was suspended in $\mathrm{CHCl}_{3}$ by sonication and filtered. Sonication and filtration were repeated twice more to ensure removal of all excess reactants and byproducts. Finally, the filter cake was washed with additional $\mathrm{CHCl}_{3}$ and then dried in vacuo. Typical yields were $700-800 \mu \mathrm{g}$ of the desired product.

\section{Results and discussion}

Two routes were employed toward addition of $R_{F}$ groups to carbon nanomaterials. First, thermal decomposition of benzoyl peroxide was used to initiate alkyl radical formation from perfluoroalkyl iodides. Radicals thus generated were used to alkylate SWNTs as well as graphene (Scheme 1). The second route, applied only to functionalize graphene flakes, was initiated by UV photolysis of perfluorodecyl iodide (Scheme 2). Perfluoroalkylated graphenes resulting from the UV- and peroxide-initiated reactions are not equivalent products, having been derivatized, respectively, with alkyl chains of 10 or 12 carbon atoms. Further, UV initiation results in derivatization with fully fluorinated decyl chains, whilst peroxideinitiated products are functionalized with dodecyl chains having two non-fluorinated carbons. The UV and peroxide reactions used microcrystalline and expanded graphite starting materials, respectively.

\subsection{Perfluorododecyl SWNTs ( $\mathrm{R}_{\mathrm{F}}$-DD-SWNTs)}

The FTIR spectra of $\mathrm{R}_{\mathrm{F}}$-DD-SWNTs contain bands in the range of $1300-900 \mathrm{~cm}^{-1}$ assignable to $v(\mathrm{C}-\mathrm{F})$ stretching modes (Fig. S-1). While this is consistent with the presence of fluorinated alkyl substituents, it does not confirm covalent attachment. FTIR spectra of $R_{F^{-}}$ DD-SWNTs produced by peroxide initiation or UV photolysis are indistinguishable. The XPS spectrum of $\mathrm{R}_{\mathrm{F}}$-DD-SWNTs indicates fluorine at an atomic 

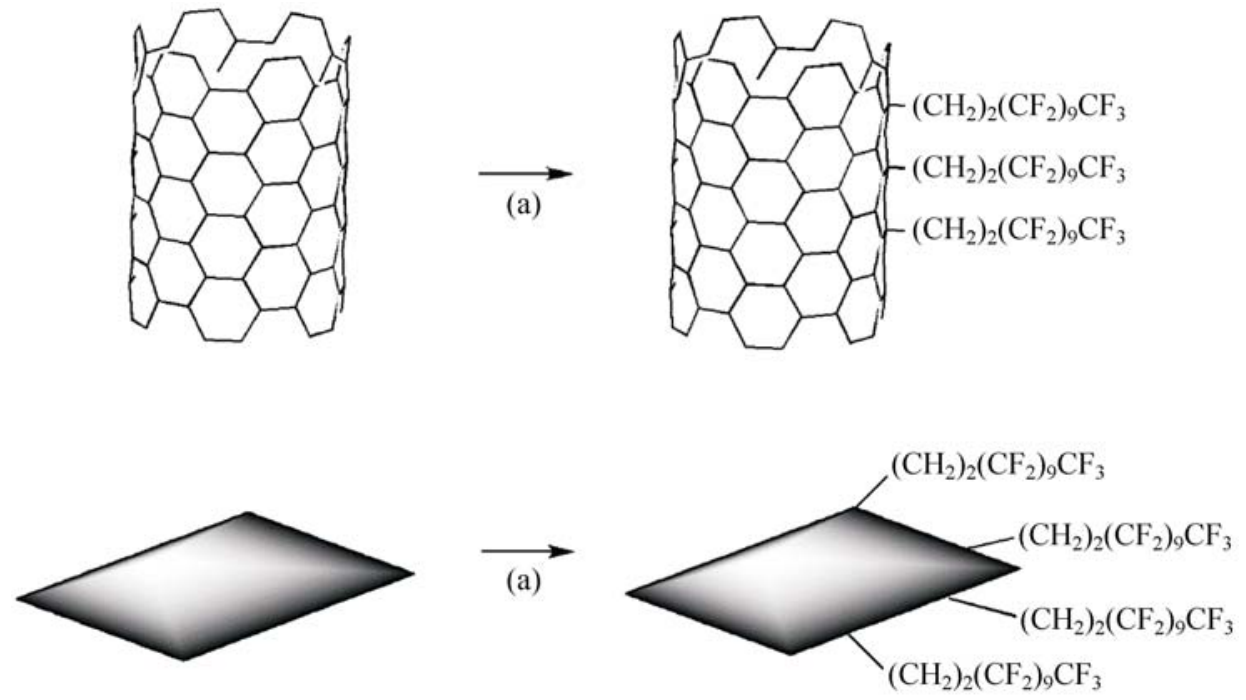

Scheme 1 Perfluorododecylated SWNTs $\left(\mathrm{R}_{\mathrm{F}}-\mathrm{DD}-\mathrm{SWNTs}\right)$ and graphene $\left(\mathrm{R}_{\mathrm{F}}-\mathrm{DD}-\mathrm{G}\right)$. (a) $\mathrm{ODCB}, \mathrm{CF}_{3}\left(\mathrm{CF}_{2}\right)_{9}\left(\mathrm{CH}_{2}\right)_{2} \mathrm{I}$, benzoyl peroxide, $80{ }^{\circ} \mathrm{C}, 24 \mathrm{~h}$
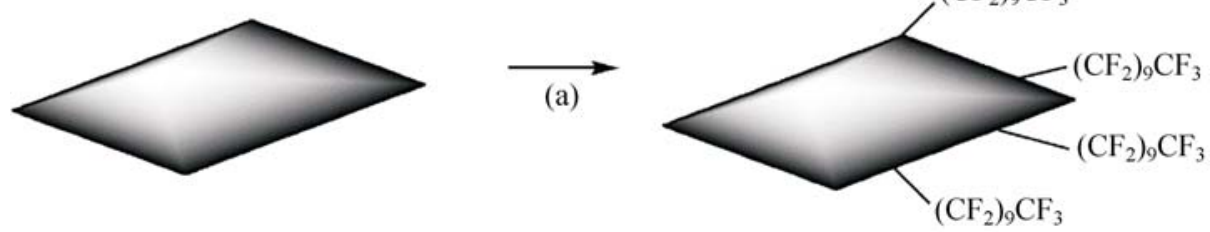

Scheme 2 Perfluorodecyl graphene ( $\left.\mathrm{R}_{\mathrm{F}}-\mathrm{D}-\mathrm{G}\right)$. (a) $\mathrm{ODCB}, \mathrm{CF}_{3}\left(\mathrm{CF}_{2}\right)_{9} \mathrm{I}, \mathrm{UV}, 8 \mathrm{~h}$

concentration of $18.5 \%$, corresponding to one functional group per 75 SWNT carbon atoms. Highresolution scans of the $\mathrm{C} 1 \mathrm{~s}$ region (Fig. 1(a)) clearly show the presence of $\mathrm{CF}_{3}(293.8 \mathrm{eV}), \mathrm{CF}_{2}(291.8 \mathrm{eV})$, $\mathrm{CH}_{2}(290.6 \mathrm{eV})$, functionalized SWNT sp3 $\mathrm{C}^{3}(286.2 \mathrm{eV})$, and pristine $\mathrm{sp}^{2}$ SWNT carbon $(284.8 \mathrm{eV})$ [17]. The $\mathrm{C} 1 \mathrm{~s}$ region also contains a peak indicative of carbon bound to oxygen at $288.9 \mathrm{eV}$, as expected, since oxidized SWNTs were used to prepare the final product. XPS of the F 1s region (Fig. 1(b)) shows a single peak centered at $688.9 \mathrm{eV}$ (in agreement with literature values for PTFE) with a small shoulder at $685.5 \mathrm{eV}$, indicative of $\mathrm{F}-\mathrm{CFCH}_{2}$ [20].

The TGA of $\mathrm{R}_{\mathrm{F}}$-DD-SWNTs in Ar shows a mass loss of $45 \%$ over the range $200-800{ }^{\circ} \mathrm{C}$, which if due solely to the perfluorododecyl group would indicate one

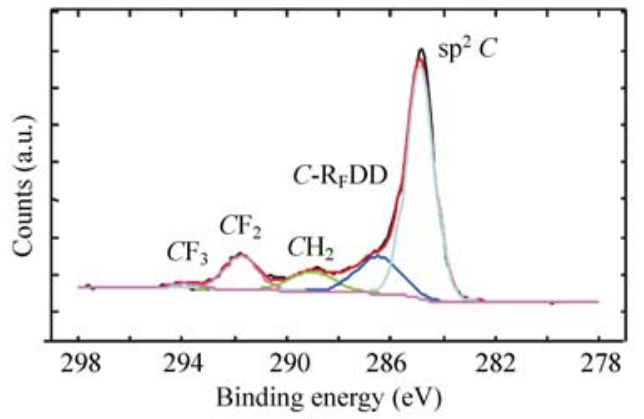

(a)

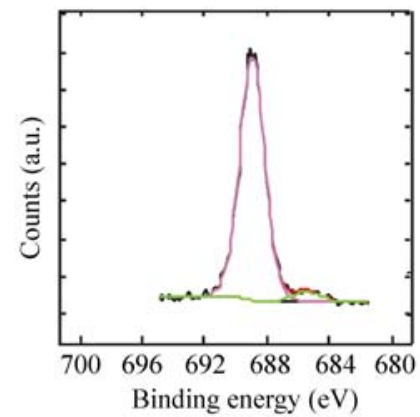

(b)

Figure 1 C 1s (a) and F 1s (b) XPS spectra of $R_{F}$-DD-SWNTs 
perfluorododecyl group per 55 SWNT carbons. This is higher than the 1:75 ratio determined by XPS (see above). There are two possible explanations for this disparity. First, etched SWNTs contain oxygen functional groups that contribute to the mass loss. Taking the oxygen content in the $\mathrm{R}_{\mathrm{F}}$-DD-SWNTs (16 atom\%) into account, results in a calculated $\mathrm{R}_{\mathrm{F}}$-DD:SWNT carbon ratio of 1:90, i.e., slightly closer to the XPS results. Second, some phenylation occurs when OCDB-generated phenyl radicals attack SWNTs directly, rather than initiating perfluoroalkyl radicals; however, the C1s XPS spectrum does not indicate significant aromatic $\mathrm{C}-\mathrm{H}$ substitution.

The Raman spectrum of $\mathrm{R}_{\mathrm{F}}$-DD-SWNTs is shown in Fig. 2. The increase in the D-band intensity (as compared to that for unreacted SWNTs) is indicative of $\mathrm{sp}^{3}$ carbon formation and consistent with the covalent attachment of perfluorododecyl groups. We have previously demonstrated [18] that the Raman $D: G$ ratio is not always an accurate measure of the extent of substitution, since functional group

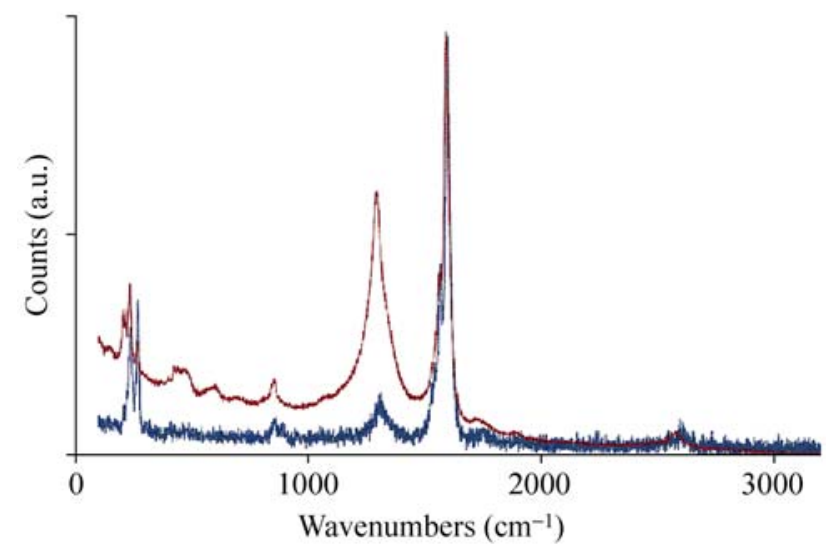

Figure 2 Raman spectrum (785 nm excitation) of $\mathrm{R}_{\mathrm{F}}$-DD-SWNTs (red) in comparison with that of the SWNT starting material (blue) distribution is also a factor in determining the $D: G$ ratio. However, the increased D:G ratio ( 0.37 from 0.07$)$ is consistent with additional covalent functionalization.

AFM of $R_{F}$-DD-SWNTs (Fig. 3) shows the presence of mostly individual functionalized SWNTs, having heights that average $1.4 \mathrm{~nm}$. There are a few small bundles present with heights of up to $4 \mathrm{~nm}$. By comparison, typical purified SWNTs are only present in bundles with heights of $20 \mathrm{~nm}$ or more. Individual purified HiPco SWNTs (debundled via surfactants) have diameters of $0.8-1.2 \mathrm{~nm}$. Line scan analysis along the length of $\mathrm{R}_{\mathrm{F}}$-DD-SWNTs shows areas of functionalization that are higher $(0.2-0.3 \mathrm{~nm})$ than unfunctionalized regions.

Our initial goal was to add perfluorinated groups to oxidatively etched SWNTs in order to suspend the products in fluorinated solvents. We found, however, that $\mathrm{R}_{\mathrm{F}}$-DD-SWNTs showed no dispersability in perfluorohexane. After attempting to suspend the material in various polar and nonpolar solvents, also without success, we noticed that the $\mathrm{R}_{\mathrm{F}}$-DD-SWNTs remained well suspended in $\mathrm{CHCl}_{3}$ during workup. In fact, suspensions of $\mathrm{R}_{\mathrm{F}}$-DD-SWNTs in $\mathrm{CHCl}_{3}$ remain stable for over one year at a concentration of $0.25 \mathrm{mg} / \mathrm{mL}$. This discovery led us to apply the same chemistry to graphene nanosheets.

\subsection{Perfluorododecyl graphene ( $\left.R_{\mathrm{F}}-\mathrm{DD}-\mathrm{G}\right)$}

XPS indicates that perfluorododecylated graphene flakes $\left(R_{F}-D D-G\right)$ contain 23.4 atom \% fluorine, indicating ca. 1 substituent per 53 carbon atoms. The $\mathrm{C} 1 \mathrm{~s}$ and $\mathrm{F} 1 \mathrm{~s}$ spectral regions are very similar to those of $\mathrm{R}_{\mathrm{F}}$-DD-SWNTs, including evidence of the presence of $\mathrm{C}$ bound to $\mathrm{O}$ (Fig. 4). Some oxidation of graphite is expected as a result of thermal expansion

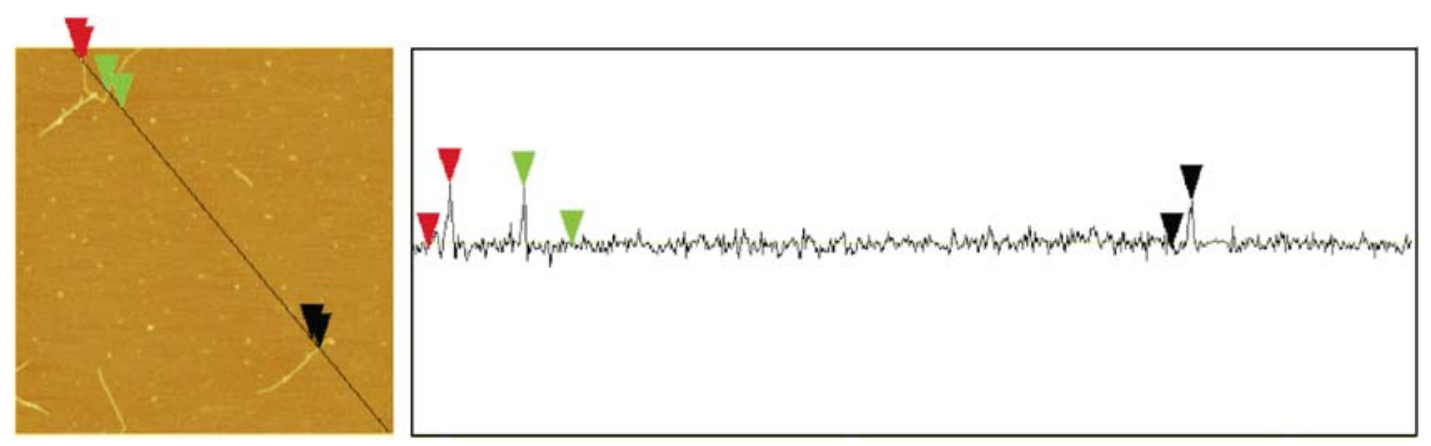

Figure 3 Representative tapping mode AFM image $(2 \mu \mathrm{m} \times 2 \mu \mathrm{m})$ and height profile of $\mathrm{R}_{\mathrm{F}}$-DD-SWNTs (height profile scale $10 \mathrm{~nm}$ ) 
during processing.

The Raman spectrum of $\mathrm{R}_{\mathrm{F}}-\mathrm{DD}-\mathrm{G}$ shows a $\mathrm{D}: \mathrm{G}$ ratio of 0.10 compared to 0.03 for the expanded graphite starting material (Fig. 5) which is indicative of covalent functionalization. This is much lower than the ratio enhancement seen for $\mathrm{R}_{\mathrm{F}}$-DD-SWNTs. Again, it should be remembered that the Raman D:G ratio depends largely on functional group distribution. It is known that functionalization of graphene flakes occurs preferentially along plate edges as opposed to the basal plane $[19,20]$, and perimeter functionalization is expected to cause less D band enhancement [21]. Raman spectroscopy was also performed on the graphite material remaining after TGA thermolysis of $\mathrm{R}_{\mathrm{F}}$-DD-G under Ar. This residue showed a reduction of the D:G ratio to 0.06 , indicating nearly complete restoration of the graphitic framework.

TGA of $R_{F}-D D-G$ showed a mass loss of $56 \%$, indicating the presence of one functional group per ca. 35 graphite carbon atoms. This is in very good

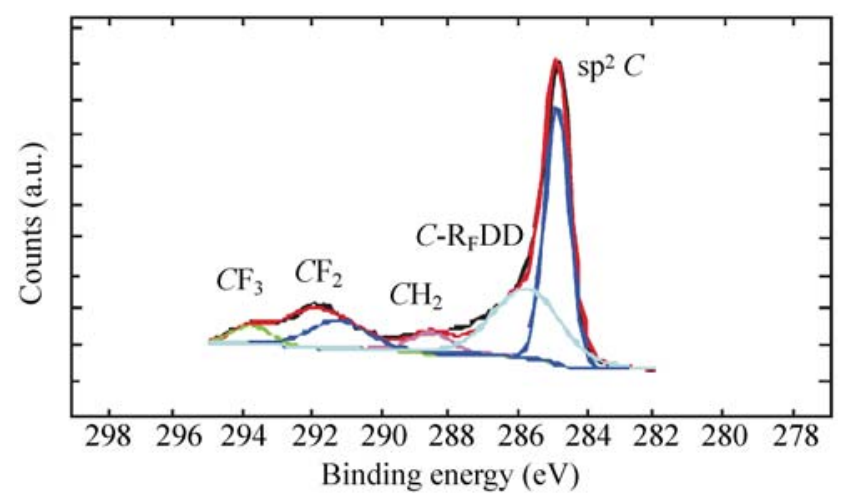

Figure 4 C 1s XPS spectrum of $R_{F}-D D-G$

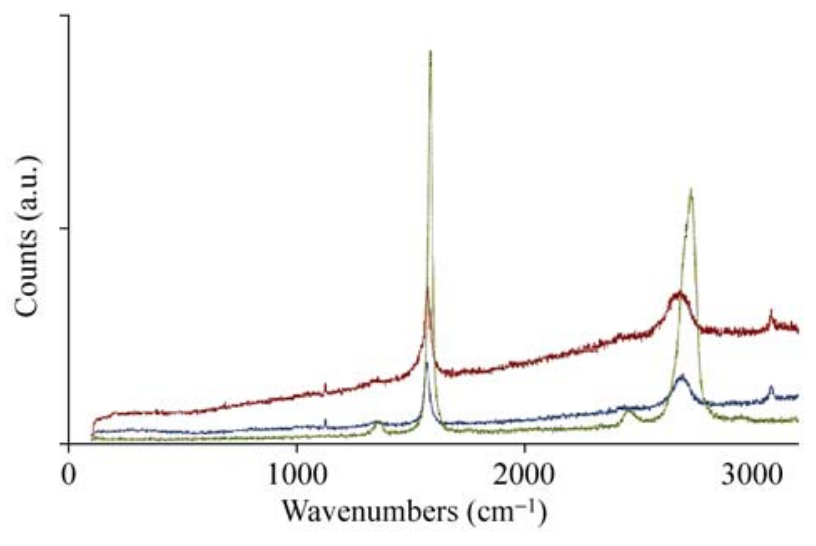

Figure 5 Raman spectra (514 nm excitation) of starting graphite (green), $\mathrm{R}_{\mathrm{F}}$-DD-G (blue), and post-thermolysis residue (red) agreement with XPS data, as well as being within range of values reported by Billups for similar graphene materials prepared by reductive alkylation [22,23]. FTIR spectra of $R_{F}$-DD-G are nearly identical to those of $\mathrm{R}_{\mathrm{F}}$-DD-SWNTs, again containing peaks in the range $1300-900 \mathrm{~cm}^{-1}$ representing C-F stretching modes.

AFM images of $\mathrm{R}_{\mathrm{F}}-\mathrm{DD}-\mathrm{G}$ indicate the presence of small nanoplatelets. The thinnest of these are 5-7 nm, with the remainder being between 15 and $20 \mathrm{~nm}$ in thickness. The lateral dimensions of the nanosheets range between 50 and $200 \mathrm{~nm}$ (Fig. 6). Additionally, areas of functionalization are visible as globular materials on the graphene flakes.

\subsection{Perfluorodecyl graphene $\left(\mathrm{R}_{\mathrm{F}}-\mathrm{D}-\mathrm{G}\right)$}

XPS of UV-produced perfluorodecyl graphene $\left(R_{\mathrm{F}}-\mathrm{D}-\mathrm{G}\right)$ shows a fluorine content of $35 \%$, corresponding to 1 substituent per 29 carbon atoms. This is in good agreement with the value for the peroxide-initiated product. The $C 1$ s region of the spectrum of $R_{F}-D-$ graphene is essentially the same as that for $R_{F}-D D-G$ with one exception: there is a small, unidentified shoulder at $282.7 \mathrm{eV}$ (Fig. 7). This peak is in the region expected for carbides or organometallic carbon. However, survey scans showed no metals are present.

Raman spectra of $R_{F}-D-G$ yielded no useful information. The D:G ratio of the product is essentially unchanged (ca. 0.15) from that in the starting material.

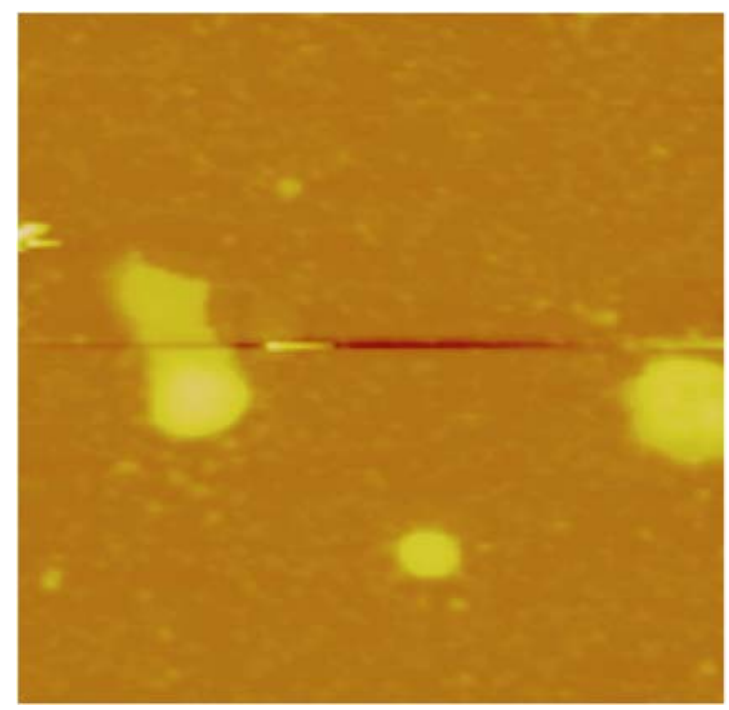

Figure 6 Representative tapping mode AFM image $(1 \mu \mathrm{m} \times$ $1 \mu \mathrm{m})$ of $\mathrm{R}_{\mathrm{F}}-\mathrm{DD}-\mathrm{G}$ 


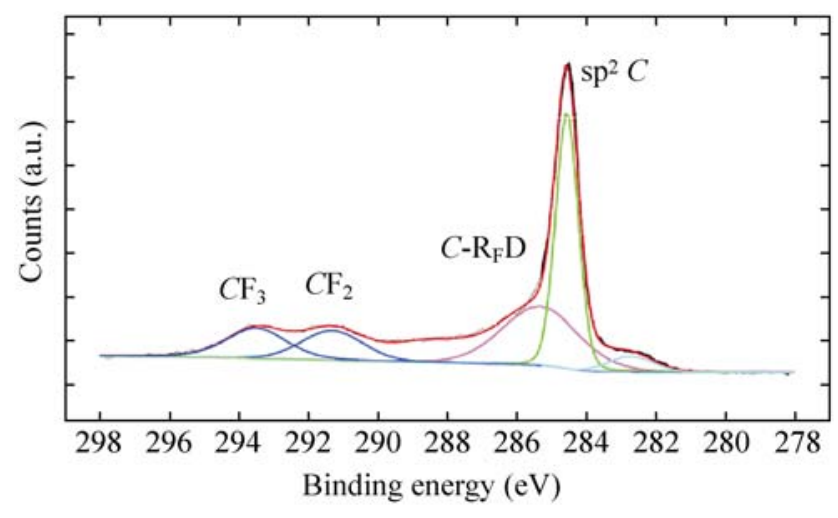

Figure 7 C 1s XPS spectrum of $R_{F}-D-G$

This is explained by the influence of edges on D-band intensity. Microcrystalline graphite consists of much smaller flakes and therefore has a larger proportion of edges. TGA of $\mathrm{R}_{\mathrm{F}}$-D-G showed a mass loss of $56 \%$, indicating a similar degree of functionalization to $\mathrm{R}_{\mathrm{F}}$-DD-G (1 $\mathrm{R}_{\mathrm{F}}$ group per 34 graphite carbons). $\mathrm{R}_{\mathrm{F}}$-Dgraphene appears to be much better exfoliated than the dodecyl product; AFM shows that nearly all the flakes are thinner than $5 \mathrm{~nm}$ (Fig. S-2), while TEM images (and associated FFT) are consistent with single or few-layer sheets (Fig. 8 and Fig. S-3).

The improved exfoliation is likely due to the difference in the graphite starting material rather than in the alkylation chemistry. Generally, ODCB dispersions of microcrystalline graphite are better exfoliated than those of expanded graphite.

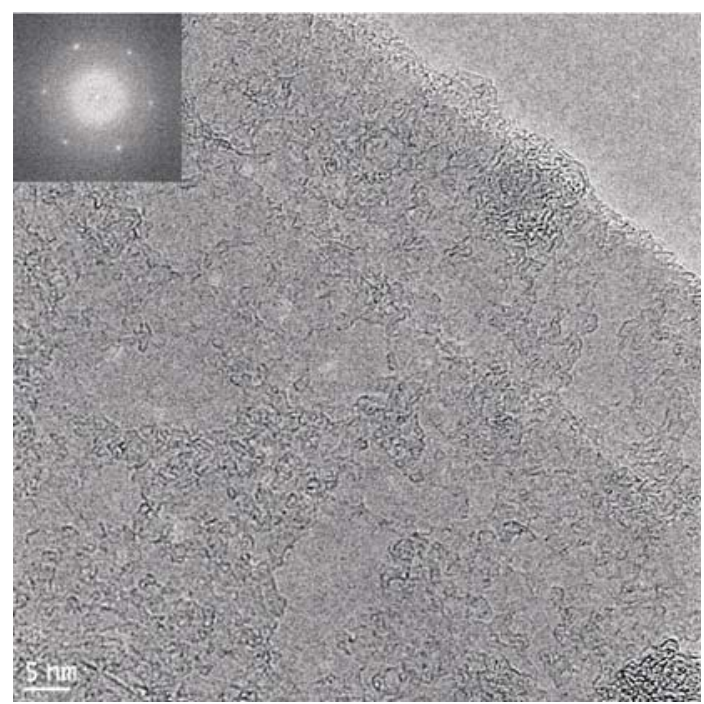

Figure 8 Representative TEM image, and associated FFT (inset), of $\mathrm{R}_{\mathrm{F}}-\mathrm{D}-\mathrm{G}$

\section{Conclusions}

We have successfully functionalized SWNTs and graphene with perfluoroalkyl groups using either thermal decomposition of benzoyl peroxide or UV photolysis to initiate radical addition in a one-pot reaction. As these perfluorinated nanocarbon hybrid materials have potential application as lubricants or polymer additives, the mechanical, tribological, and thermal properties of polymer dispersions of the reaction products are presently being examined.

\section{Acknowledgements}

Financial support for this work was provided by Wright-Patterson Air Force Laboratory and the AFOSR, the Robert A. Welch Foundation, the Advanced Energy Consortium and the Department of Energy's Hydrogen Storage Program.

Electronic Supplementary Material: Supplementary material is available in the online version of this article at http://dx.doi.org/10.1007/s12274-010-1007-3 and is accessible free of charge.

\section{References}

[1] Bahr, J. L.; Tour, J. M. Covalent chemistry of single-wall carbon nanotubes. J. Mater. Chem. 2002, 12, 1952-1958.

[2] Geim, A. K.; Novoselov, K. S. The rise of graphene. Nat. Mater. 2007, 6, 183-191.

[3] Dyke, C. A.; Tour, J. M. Covalent functionalization of single-walled carbon nanotubes for materials applications. $J$. Phys. Chem. A 2004, 108, 11151-11159.

[4] Adams, D. J.; Dyson, P. J.; Tavener, S. J. In Chemistry in Alternative Reaction Media. Wiley: Sussex, 2004; pp. 57-71.

[5] Hamilton, C. E.; Ogrin, D.; McJilton, L.; Moore, V. C.; Anderson, R.; Smalley, R. E.; Barron, A. R. Functionalization of SWNTs to facilitate the coordination of metal ions, compounds and clusters. Dalton Trans. 2008, 2937-2944.

[6] Smalley, R. E.; Li, Y.; Moore, V. C.; Price, B. K.; Colorado, R. Jr.; Schmidt, H. K.; Hauge, R. H.; Barron, A. R.; Tour, J. M. Single wall carbon nanotubes amplification: En route to a type-specific growth mechanism. J. Am. Chem. Soc. 2006, 128, 15824-15829.

[7] Fagan, P. J.; Krusic, P. J.; McEwen, C. N.; Lazar, J.; Parker, D. H.; Herron, N.; Wasserman, E. Production of perfluoroalkylated nanospheres from buckminsterfullerene. 
Science 1993, 262, 404-07.

[8] Holzinger, M.; Vostrowsky, O.; Hirsch, A.; Hennrich, F.; Kappes, M.; Weiss, R.; Jellen, F. Sidewall functionalization of carbon nanotubes. Angew. Chem. Int. Ed. 2001, 40, 4002-4005.

[9] Voggu, R.; Biswas, K.; Govindaraj, A.; Rao, C. N. R. Use of fluorous chemistry in the solubilization and phase transfer of nanocrystals, nanorods, and nanotubes. J. Phys. Chem. B 2006, 110, 20752-20755.

[10] Pulikkathara, M. X.; Kuznetsov, O. V.; Peralta, I. R. G.; Wei, X.; Khabashesku, V. N. Medium density polyethylene composites with functionalized carbon nanotubes. Nanotechnol. 2009, 20, 195602-195605.

[11] Ying, Y. M.; Saini, R. K.; Liang, F.; Sadana, A. K.; Billups, W. E. Functionalization of carbon nanotubes by free radicals. Org. Lett. 2003, 5, 1471-1473.

[12] Liang, F.; Beach, J. M.; Rai, P. K.; Guo, W. H.; Hauge, R. H.; Pasquali, M.; Smalley, R. E.; Billups, W. E. Highly exfoliated water-soluble single-walled carbon nanotubes. Chem. Mater. 2006, 18, 1520-1524.

[13] Armarego, W. L. F.; Perrin, D. D. In Purification of Laboratory Chemicals. Butterworth-Heinemann: Oxford, 4th Ed., 1997; p. 105.

[14] Chiang, I. W.; Brinson, B. E.; Huang, A. Y.; Willis, P. A.; Bronikowski, M. J.; Margrave, J. L.; Smalley, R. E.; Hauge, R. H. Purification and characterization of single-wall carbon nanotubes (SWNTs) obtained from the gas-phase decomposition of CO (HiPco process). J. Phys. Chem. B 2001, 105, 8297-8301.

[15] Ziegler, K. J.; Gu, Z. N.; Peng, H. Q.; Flor, E. L.; Hauge, R. H.;
Smalley, R. E. Controlled oxidative cutting of single-walled carbon nanotubes. J. Am. Chem. Soc. 2005, 127, 1541-1547.

[16] Hamilton, C. E.; Lomeda, J. R.; Sun, Z. Z.; Tour, J. M.; Barron, A. R. High-yield organic dispersions of unfunctionalized graphene. Nano Lett. 2009, 9, 3460-3462.

[17] NIST XPS spectral database, http://srdata.nist.gov/xps/ (access Oct 5, 2009).

[18] Zhang, L.; Zhang, J.; Schmandt, N.; Cratty, J.; Khabashesku, V. N.; Kelly, K. F.; Barron, A. R. AFM and STM characterization of thiol and thiophene functionalized SWNTs: Pitfalls in the use of gold nanoparticles to determine the extent of side-wall functionalization in SWNTs. Chem. Commun. 2005, 5429-5430.

[19] Lerf, A.; He, H. Y.; Forster, M.; Klinowski, J. Structure of graphite oxide revisited. J. Phys. Chem. B 1998, 102, 4477-4482.

[20] Chattopadhyay, J.; Mukherjee, A.; Hamilton, C. E.; Kang, J. -H.; Chakraborty, S.; Guo, W. H.; Kelly, K. F.; Barron, A. R.; Billups, W. E. Graphite epoxide. J. Am. Chem. Soc. 2008, 130, 5414-5415.

[21] Ferrari, A. C.; Meyer, J. C.; Scardaci, V.; Casiraghi, C.; Lazzeri, M.; Mauri, F.; Piscanec, S.; Jiang, D.; Novoselov, K. S.; Roth, S.; Geim, A. K. Raman spectrum of graphene and graphene layers. Phys. Rev. Lett. 2006, 97, 187401.

[22] Chakraborty, S.; Guo, W.; Hauge, R. H.; Billups, W. E. Reductive alkylation of fluorinated graphite. Chem. Mater. 2008, 20, 3134-3136.

[23] Chakraborty, S.; Chattopadhyay, J.; Guo, W. H.; Billups, W. E. Functionalization of potassium graphite. Angew. Chem. Int. Ed. 2007, 46, 4486-4488. 